\title{
Designing Incentive Compatible Contracts for Effective Demand Management
}

\author{
Murat Fahriog̃lu, Student Member, IEEE and Fernando L. Alvarado, Fellow, IEEE
}

\begin{abstract}
Demand relief from customers can help a utility (or any "Load Serving Entity") solve a variety of problems. There exist all sorts of different demand management programs that utilities use. A critical issue is the incentive paid to the customer to participate in demand management programs and provide load relief. The utility has to design cost effective yet attractive demand management contracts. The main goal is to get load relief when needed, and to do so in a cost effective way. Customers sign up for programs when the benefits they derive in the form of up front payments, demand discounts and interruption payments exceed their cost of interruption. In order to design such contracts, mechanism design with revelation principle is adopted from Game Theory and applied to the interaction between a utility and its customers. The idea behind mechanism design is to design an incentive structure that encourages customers to sign up for the right contract and reveal their true value of power (and thus, the value of power interruptibility).
\end{abstract}

Index Terms-Demand management, game theory, load curtailment, load interruption, mechanism design, system security.

\section{INTRODUCTION}

$\mathbf{T}$ HE INCREASED penetration of backup generation [2] and energy management systems opens the door for more creative means for integrating demand management into utility operations. By explicitly examining customer outage costs [4], [2] and analyzing their load behavior, it is possible for a utility (or any "Load Serving Entity") to design different kinds of demand management programs and attract customers to help in case of emergencies in return for an incentive fee [7]. Because a utility can only estimate the outage costs to a customer, it is difficult for a utility to know how much incentive to offer in order to attract customers to curtail or interrupt their load. The main theme of this paper is to design cost effective demand management programs that do not require the knowledge of customer outage costs, but rather use Game Theory [8] to design optimal curtailment programs. The process of designing contracts that attain this objective is called mechanism design with revelation principle. The mechanism (or contract offered by the utility) makes sure that the utility's benefit is maximized and that customers are compensated sufficiently to participate voluntarily. A new general formulation is developed and illustrated by means of an example. The paper also combines the economic aspects. of contracts with power system sensitivity analysis. Sensitivity methods attribute value to power interruptibility at every location in the grid. Thus, contracts can be customized by location.

Manuscript received April 12, 1999; revised August 31, 1999. This work was supported by the Energy Center of Wisconsin.

The authors are with the Department of Electrical and Computer Engineering, The University of Wisconsin-Madison.

Publisher Item Identifier S 0885-8950(00)10356-6.
This approach to demand management is likely to prove more useful than the traditional "lower rates" interruptible contract approach because it more clearly lines up the interests of diverse customer types with diverse locational utility needs.

\section{MeChanism DeSIGN}

Mechanism design and the revelation principle are key concepts used in nonlinear pricing. They are explained in detail in, among other places, [8] and [13]. Mechanism design is a powerful tool that helps a principal (in this case, the utility), with no private information about its customers, decide in an optimal way how much to buy from (or sell to) its customers and at what price. The revelation principle [9], [5], [14] is used to simplify the problem. The mechanism (or contract offer structure) can be designed so that customers wishing to maximize their own total benefit are encouraged to reveal their true valuation of power interruptions.

The mechanism has two kinds of output: a decision vector (amounts to buy or sell) and a vector of monetary transfers from the principal to each customer. This theory is applied to the interaction between a utility and its customers. In order to better understand these issues, it is desirable to understand nonlinear pricing.

\section{A. Nonlinear Pricing in General}

Consider that a customer values a product according to a declining marginal benefit as a function of amount consumed (denoted by $q$ ). Let the marginal benefit be described by:

$$
b(q)=\theta\left(b_{0}-s q\right)
$$

where $\theta$ (normalized as $0<\theta<1$ ) is a parameter that depends on the customer; it represents the "customer type." Customers with the same $\theta$ have the same cost-quantity characteristics. $b_{0}$ is the value of the very first unit of commodity consumed, and $s$ is how rapidly the marginal value of additional consumption declines. Fig. 1 illustrates the marginal benefit function for $b_{0}=$ $1, s=1$ and two values of $\theta$.

The total benefit $B$ is the integral of this marginal benefit. For the type of benefit function assumed above, the following is the total benefit:

$$
B(\theta, q)=\theta b_{0} q-\frac{1}{2} s \theta q^{2}
$$

Fig. 2 illustrates the total benefit curves for each of the two customer types.

A principal needs to design a nonlinear pricing scheme to sell this product. Let the per unit cost of producing be $u$. Assume that the principal elects to consider only two types of customers 


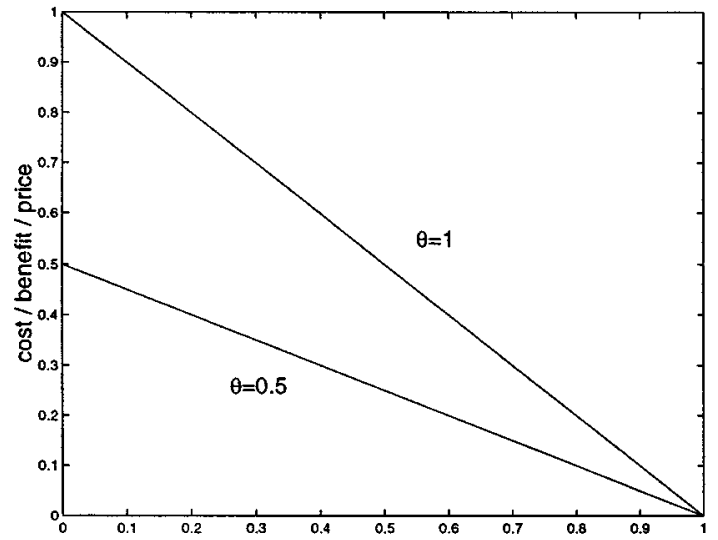

Fig. 1. Marginal benefit for two customer types.

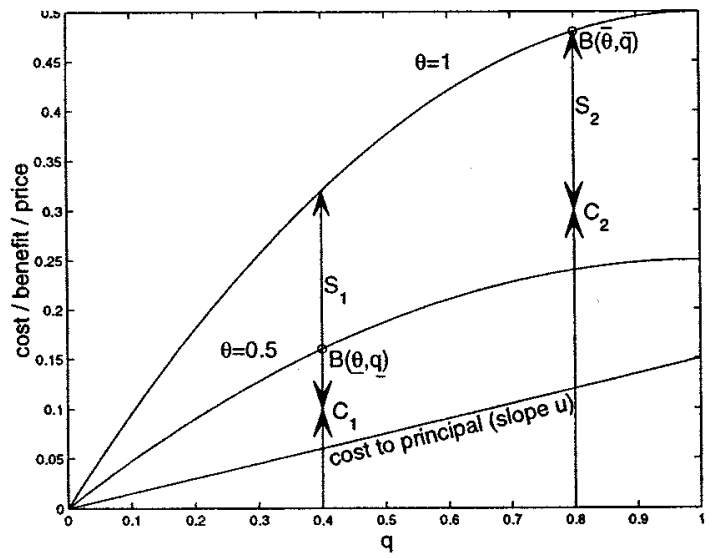

Fig. 2. Total benefit, cost to producer and consumption levels for two customer types.

it wishes to sell to, a small customer to which it wishes to sell quantity $\underline{q}$ and a large customer to which it wishes to sell a quantity $\bar{q}$, with $\underline{q}$ and $\bar{q}$ yet to be determined $(\underline{q}<\bar{q})$. The cost to produce $\underline{q}$ is $u \underline{q}$. Likewise, the cost to produce $\bar{q}$ is $u \bar{q}$. The straight line defining these (and any other) production costs is also illustrated in Fig. 2. The principal wishing to sell at a profit selects price/quantity points that lie at or above this line. Let $C_{1}$ be the price chosen for quantity $q$ and $C_{2}$ be the selected sale price for quantity $\bar{q}$ (as shown in Fig. 2). Clearly, a principal can hope to sell to the small customer (with type $\underline{\theta}$ ) only if $B(\underline{\theta}, \underline{q})>C_{1} \underline{q}$ and it can hope to sell to the large customer (with type $\bar{\theta}$ ) only if $B(\bar{\theta}, \bar{q})>C_{2} \bar{q}$. This condition is called the individual rationality constraint.

A more subtle constraint exists: if the principal were to always charge prices close to $B(\bar{\theta}, q)$, the small consumer would be unable to buy, since this would be done at a loss. Assume that there is at least one price/quantity offering equal to or below curve $B(\underline{\theta}, q)$ (but above $u q$ ). This is, indeed, the case for the $\left(C_{1}, q\right)$ offering. If the large consumer were to choose a small amount of consumption $(q)$, its net benefit (total benefit- $C_{1} q$ ) would be the segment illustrated as $S_{1}$. If, on the other hand, it were to consume the large amount $(\bar{q})$, its net benefit would be segment $S_{2}$. It seems reasonable that if the large customer can derive more benefit by consuming less (that is, if $S_{1}>S_{2}$ ), it is going to consume less. This is almost never desirable to the principal, as it results in highly suboptimal conditions. Thus, we require that pricing be such that $S_{1} \leq S_{2}$ for the larger customer. This condition is called the incentive compatibility constraint. Fig. 2 illustrates a case that violates this condition, and thus encourages the customer to "lie." It can be shown mathematically that optimality requires that the lower consumption/price point be determined by the individual rationality constraint, and that the upper price be determined by a binding incentive compatibility constraint.

If only the large customer existed, optimality would be attained when $(d B(\bar{\theta}, q)) /(d q)=u$. If only the small customer existed, it would be optimal to select the situation where $(d B(\underline{\theta}, q)) /(d q)=u$. It is the role of mechanism design to design pricing structures so that optimality is attained where there is a mix of customers, and when there is uncertainty about the mix.

\section{B. Contract Design}

The cost of power curtailment to a customer depends on both the customer and the amount interrupted. We assume, at least initially, that the $\operatorname{cost} c(\theta, x)$ to a customer of type $\theta$ of curtailing $x$ MW is:

$$
c(\theta, x)=K_{1} x^{2}+K_{2} x-K_{2} x \theta .
$$

Here $\theta$ is a continuous variable describing the customer type. ${ }^{1}$ It can also be called the customer preference parameter. The " $-K_{2} x \theta$ " term is included so that different values of $\theta$ lead to different values of $\partial c / \partial x$ (marginal cost for the customer). Notice that, as $\theta$ increases the marginal cost decreases. That is, $\theta$ has effectively been used to "sort" the customers from "least willing" to "most willing" to shed load. This form of the cost function suggests that the customer with the lowest $\theta$ will have the highest marginal cost and hence the lowest marginal benefit. This provides a good way of modeling the willingness of each customer to shed load by way of $\theta$.

For the sake of simplicity, we assume that $K_{1}$ and $K_{2}$ are known to be $1 / 2$ and 1 , respectively. These assumptions do not affect the fundamental concepts to be considered here, since they amount to simple scaling. Customers of different types value interruptions differently. Although (3) gives an expression for the cost of an interruption to a customer, the parameter $\theta$ in this equation is not known to the utility.

Another assumption concerns the probability distribution of $\theta$ [denoted by $f(\theta)$ ]. Two possibilities are:

1) The complete set of customer types can be characterized by allowing $\theta$ to vary from 0 to 1 , and there is an equal probability that the customer will be of any of these types [that is, $\theta$ is a random variable with a uniform distribution in the interval $(0,1)]$.

2) The parameter $\theta$ can take discrete values, each with a presumed probability. Two discrete values of $\theta$ represent the simplest such scenario.

The probability distributions associated with $\theta$ are subjective probabilities. The utility need not know which, if any, of the distributions is correct. The value of $\theta$ is private information of the

\footnotetext{
${ }^{1} \theta$ allows us to model multiple customer types by giving each type of customer a different cost of curtailment.
} 
customer and is unknown to the utility. Having a subjective estimate of the customer types it is dealing with, the utility develops an incentive function $y(x)$ to indicate how much it is willing to pay for a given amount of curtailment.

Customers self-select the amount of curtailment they wish to be subjected to, based on an inspection of the incentive function offered to them. Clearly, customers, will not choose to be curtailed unless they see a net positive benefit. A customer's benefit function is:

$$
V_{1}(\theta, x, y)=y-\frac{1}{2} x^{2}-x+\theta x .
$$

In the absence of an initial sign-up incentive, ${ }^{2}$ in order for a customer to elect to participate in a program, it is necessary that $V_{1} \geq 0$, that is they must see a benefit to the curtailment.

This quadratic, or any other arbitrary cost function, needs to satisfy the sorting (or "single crossing") condition [8] in order to make mechanism design work. If the customers are sorted from least willing (to shed load) to most willing the sorting condition dictates that

$$
\frac{\partial}{\partial \theta}\left(\frac{\partial c}{\partial x}\right)<0
$$

Likewise, if the customers are sorted from most willing to least willing the condition becomes

$$
\frac{\partial}{\partial \theta}\left(\frac{\partial c}{\partial x}\right)>0
$$

Whether one sorts customers according to increasing or decreasing willingness is a matter of preference and is irrelevant (we will assume increasing willingness). The important issue is that the outage cost function be monotonic in $\theta$ and nondecreasing in $x$. The function in (4) satisfies this property.

Under stressed conditions it is expensive for the utility to deliver power to certain locations. The utility can compute the value of not delivering power to a certain customer. This "value of power interruptibility" is parameterized by $\lambda$. The value of $\lambda$ can be computed using existing efficient optimal power flow routines [6], [12]. Knowing $\lambda$ enables the utility to define their own benefit function for a curtailment at a specific location:

$$
V_{2}(\theta, \lambda)=\lambda x(\theta)-y(\theta)
$$

where $\lambda$ is in dollars per MW not delivered to a customer. The objective of the utility is to maximize the utility benefit function.

$$
\max _{x, y} \int_{0}^{1}[\lambda x(\theta)-y(\theta)] f(\theta) d \theta
$$

such that,

$y(\theta)-\frac{1}{2} x^{2}(\theta)-x(\theta)+\theta x(\theta) \geq 0$

$y(\theta)-\frac{1}{2} x^{2}(\theta)-x(\theta)+\theta x(\theta) \geq y(\hat{\theta})-\frac{1}{2} x^{2}(\hat{\theta})-x(\hat{\theta})+\theta x(\hat{\theta})$

where $\hat{\theta}$ is the preference parameter of a customer if they were to report it incorrectly. If a customer picks any of the contracts

\footnotetext{
${ }^{2} \mathrm{~A}$ fixed one-time sign up incentive can be a part of the overall compensation, but it is not considered here. It would have the effect of modifying the perceived net customer benefit.
}

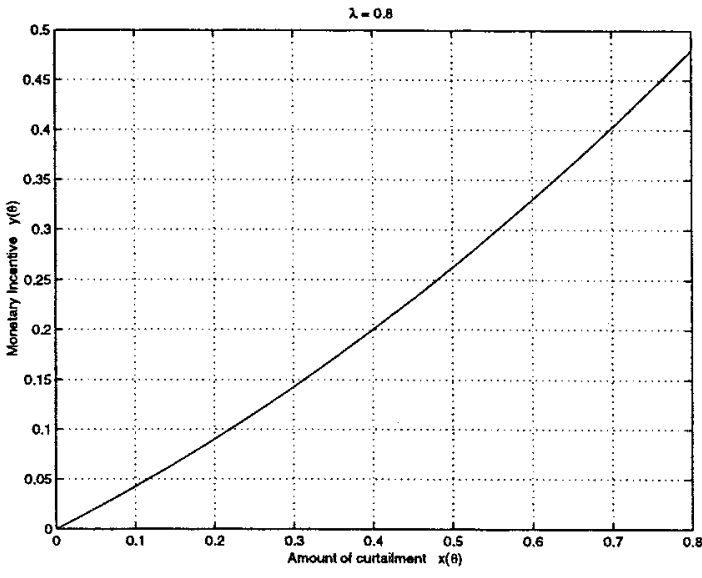

Fig. 3. Designed contracts

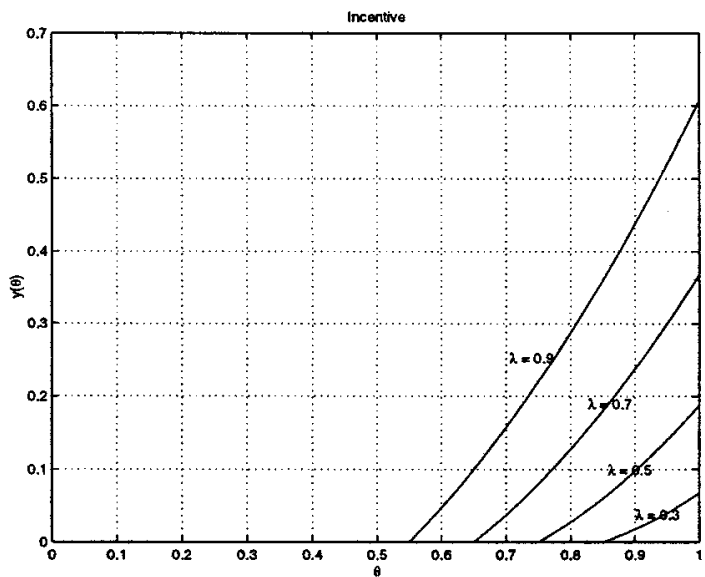

Fig. 4. Normalized incentive function vs. $\theta$.

that is not specifically designed for them, ${ }^{3}$ they pose as another type of customer $(\hat{\theta})$. Constraint (7) is the individual rationality constraint which makes sure every customer is encouraged to participate, and constraint (8) is the incentive compatibility constraint which encourages the customers to tell the truth about their $\theta$ (i.e. to pick the right contract). This maximization problem could be solved by using mechanism design and the revelation principle [8]. The results are:

$$
\begin{aligned}
& x(\theta)= \begin{cases}0 & \text { if } 0 \leq \theta<1-\frac{\lambda}{2} \\
2 \theta+\lambda-2 & \text { if } 1-\frac{\lambda}{2} \leq \theta<1\end{cases} \\
& y(\theta)= \begin{cases}0 & \text { if } 0 \leq \theta<1-\frac{\lambda}{2} \\
\theta^{2}-2 \theta+2 \theta \lambda & \text { if } 1-\frac{\lambda}{2} \leq \theta \leq 1 \\
+\frac{3}{4} \lambda^{2}-2 \lambda+1\end{cases}
\end{aligned}
$$

Equations (9) and (10) define the contracts to be offered to different types of customers. Fig. 3 depicts the plot of the monetary incentive offered as a function of the curtailed amount for a given value of $\lambda$. A family of incentive functions as $\lambda$ varies is shown in Fig. 4. The number of participating customers changes as the value of $\lambda$ changes. Fig. 4 shows that the value of $\lambda$ plays a key role in determining the incentive to be paid to each customer.

\footnotetext{
${ }^{3}$ The mechanism designs a specific contract for each type of customer (i.e. for each value of $\theta$ ). $\theta$ values can be estimated from existing utility data.
} 
The role of $\theta$ is obvious since it determines the type of customer (which in turn determines their cost of a curtailment or interruption), however the role of $\lambda$ can be subtle. $\lambda$ is the parameter that brings engineering into this economic analysis. It shows that location of the customer is one of the most important aspects in this analysis. Some locations will be more costly to deliver power than others. It makes sense that the utility will want to have curtailment contracts with customers who are at expensive locations.

\section{SENSITIVITy ANALYSIS}

Sensitivity analysis can be used to determine the value of interruptible power for the utility. In [10], the authors compute the sensitivity of the loading margin of a system with respect to arbitrary parameters. If loads are the parameters, sensitivity of the loading margin can be computed with respect to each load. Let:

$$
g(x, \gamma, p)=0
$$

where

$x \quad$ is the vector of state variables,

$\gamma \quad$ is the vector of real and reactive load powers, and

$p \quad$ is the vector of loads.

If a pattern of load increase is specified with a unit vector $k$, the point of collapse method [3] can be applied to yield the left eigenvector $w$. The sensitivity of the loading margin to a change in any load is:

$$
\frac{\Delta L}{\Delta p}=L_{p}=\frac{-w g_{p}}{w g_{\gamma} k}
$$

Once we have the sensitivity of the loading margin to a change in any load, we use it to rank loads. Let $L$ be the loading margin of the system. The above formula lets us construct an expression relating changes in individual loads ( $\Delta p_{1}, \Delta p_{2}$, etc.) to changes in the security margin:

$$
\Delta L=L_{p_{1}} \Delta p_{1}+L_{p_{2}} \Delta p_{2}+\cdots+L_{p_{m}} \Delta p_{m}
$$

where $m$ is the number of loads of interest. As equation (13) suggests, the load with the highest sensitivity would help increase the loading margin the most. By using these sensitivities and the dollar per $\mathrm{kW}$ figures from the designed contracts, the utility can estimate how much it costs to increase system security:

$$
\frac{\Delta L}{\Delta S}=\frac{\Delta L}{\Delta p} \frac{\Delta p}{\Delta \$}
$$

where $\Delta \$$ is the amount the utility will spend.

Equation (14) helps determine how much it costs to increase the loading margin by curtailing one of the loads. Other kinds of sensitivities can be computed and combined with the economic analysis done in the previous section to give the utility the cost of solving specific security problems.

Whenever possible it is more efficient for markets to resolve congestion than to have side-markets for demand management. Locational Marginal Pricing (LMP) in various forms has been adopted by many markets as a means to resolve most congestion problems [15]. However, there are some situations where a pure market may be unable to resolve a serious security constraint. In these cases the use of cost effective demand management is called for. For these situations, the knowledge of margins and margin sensitivities is essential.

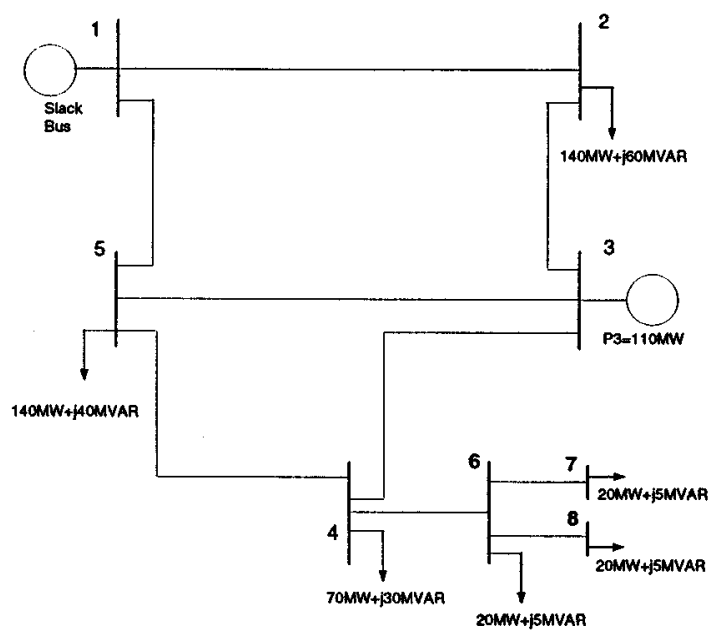

Fig. 5. Sample 8-bus system.

TABLE I

SENSITIVITY OF THE LOADING MARGIN TO VOLTAGE COLLAPSE WITH RESPECT TO EACH LOAD (LOADING DIRECTION CHOSEN AS EQUAL INCREMENTS FOR EACH LOAD)

\begin{tabular}{c|c}
\hline Load Bus & Sensitivity (MW/MW) \\
\hline 2 & -0.03 \\
\hline 4 & -0.89 \\
\hline 5 & -0.12 \\
\hline 6 & -1.48 \\
\hline 7 & -1.73 \\
\hline 8 & -1.73 \\
\hline \multicolumn{2}{|c|}{ Loading Margin $=36.18 \mathrm{MW}$} \\
\hline
\end{tabular}

\section{AN EXAMPLE}

Before demand management contracts are offered to customers, utilities need to go through a planning stage. The first step is to analyze their electric power system and identify which load locations (customers) would be most helpful in case of emergencies or anticipated problems (voltage collapse, line overloads, insufficient generation, etc.). A sensitivity analysis needs to be performed on the system to determine the most valuable loads for each type of problem. This analysis involves load forecasting and consideration of multiple scenarios and time periods. ${ }^{4}$ The example involves three stages:

1) Sensitivity analysis to determine the most valuable loads to increase loading margin to voltage collapse.

2) Game Theory analysis to determine the optimal demand management contracts.

3) Comparison of different scenarios of demand management contracts.

The example uses an 8 bus system (see Fig. 5) with 2 generators and 6 loads. Of concern is the loading margin to voltage collapse. If the load is increased equally on each load bus and only the slack generator picks up the extra load, the sensitivity of the loading margin to voltage collapse with respect to a change in each load is shown in Table I. In this example the most valuable loads are 7 and 8 . They have the highest sensitivity. If the system gets close to a voltage bifurcation point [1], [11] the utility may want to curtail a guaranteed amount of load, hence the contracts

\footnotetext{
${ }^{4}$ It is assumed that demand management contracts can vary by location, class and type of customer.
} 
TABLE II

OPtimal PoRTFOLIO OF DEMAND MANAGEMENT CONTRACTS

\begin{tabular}{c|c|c}
\hline Customer & $\begin{array}{c}\text { Amount Curtailed } \\
x\end{array}$ & $\begin{array}{c}\text { Incentive Offered } \\
y\end{array}$ \\
\hline Bus 2 & $1.00 \mathrm{MW}$ & $\$ 225.00$ \\
\hline Bus 4 & $3.50 \mathrm{MW}$ & $\$ 1620.00$ \\
\hline Bus 5 & $3.00 \mathrm{MW}$ & $\$ 975.00$ \\
\hline Bus 6 & $4.00 \mathrm{MW}$ & $\$ 2000.00$ \\
\hline Bus 7 & $5.00 \mathrm{MW}$ & $\$ 2875.00$ \\
\hline Bus 8 & $7.00 \mathrm{MW}$ & $\$ 4375.00$ \\
\hline \multicolumn{3}{|c}{ Available Relief $=24 \mathrm{MW}$} \\
\hline \multicolumn{2}{|c}{ Increase in Margin $=30 \mathrm{MW}$} \\
\hline
\end{tabular}

TABLE III

Non-Optimal Portfolio of Demand Management Contracts WITH FiXed $\lambda=0.7$ (AVERAge VAlue)

\begin{tabular}{c|c|c}
\hline Customer & $\begin{array}{c}\text { Amount Curtailed } \\
x\end{array}$ & $\begin{array}{c}\text { Incentive Offered } \\
y\end{array}$ \\
\hline Bus 2 & $1.00 \mathrm{MW}$ & $\$ 375.00$ \\
\hline Bus 4 & $3.00 \mathrm{MW}$ & $\$ 1275.00$ \\
\hline Bus 5 & $5.00 \mathrm{MW}$ & $\$ 2375.00$ \\
\hline Bus 6 & $3.00 \mathrm{MW}$ & $\$ 1275.00$ \\
\hline Bus 7 & $3.00 \mathrm{MW}$ & $\$ 1275.00$ \\
\hline Bus 8 & $5.00 \mathrm{MW}$ & $\$ 2375.00$ \\
\hline \multicolumn{3}{|c}{ Available Relief $=20 \mathrm{MW}$} \\
\hline \multicolumn{3}{|c}{ Increase in Margin $=22 \mathrm{MW}$} \\
\hline
\end{tabular}

would be designed for specific amounts of load curtailment. It is important to determine the ranking and quantitative impact of loads before the utility offers curtailment contracts. After the contracts are signed, an algorithm can be developed to check for distance to collapse and suggest the optimum curtailment order of loads when required.

After the relative value of all load locations is determined, customer attributes, designed demand management programs and the game theory formulation are used to design optimal demand management contracts ${ }^{5}$ (similar to the contracts shown in Fig. 3). If the assumptions hold, the customers will sign up for the contracts as shown in Table II. The customers at location bus 7 and 8 signed up for different contracts even though they are at equally sensitive locations. This is because the customer at bus 7 has a higher marginal cost for shedding load than the customer at bus 8 . The developed contract design formulation captures both locational and cost attributes of the customers.

The value of power interruptibility $(\lambda)$ and customer preference parameter (private information $\theta$ that helps the utility estimate customer cost) are the two critical elements of the economic analysis. In order to emphasize the importance of these values, some further tests are performed. In one simulation $\lambda$ was fixed to be 0.7 (its average value), i.e. the utility decides that the value of interrupting power at each location is the same. As shown in Table III the amount of available relief decreased and the result was also a smaller increase in the loading margin. In another simulation the utility assumes that the cost of shedding load is the same to all customers [i.e. it fixes $\theta$ at its average value $(\theta=0.8)$ ]. This also is not optimal since the available load relief and the increase in loading margin is lower than the optimal case shown in Table II. Some economic facts are

\footnotetext{
5These are "Agreed Relief" [7] contracts i.e. the customer agrees to curtail a "guaranteed" amount of load for a monetary incentive.
}

TABLE IV

DIFFERENT SCENARIOS FOR CONTRACTS

\begin{tabular}{|c|c|c|c|c|}
\hline Scenario & $\begin{array}{c}\text { Relief } \\
(\mathrm{MW})\end{array}$ & $\begin{array}{c}\uparrow \mathrm{LM} \\
(\mathrm{MW})\end{array}$ & $\begin{array}{c}\text { U. Profit } \\
(\$)\end{array}$ & $\begin{array}{c}\text { C. Profit } \\
(\$)\end{array}$ \\
\hline Optimal & 24 & 30 & 6456.00 & 2806.00 \\
\hline$\theta=0.8$ & 19 & 26 & 5681.00 & 1981.00 \\
\hline$\lambda=0.7$ & 20 & 22 & 5050.00 & 1950.00 \\
\hline
\end{tabular}

also computed for each portfolio and a comparison is made in Table IV. In the scenarios where $\lambda$ and $\theta$ are fixed to their average values, a nonoptimal portfolio of contracts is still obtained, however the results verify that the optimal portfolio of contracts help both the utility and the customer in maximizing their profit. More importantly, it indicates that the optimal portfolio maximizes the amount of available load relief and the increase in the loading margin.

Several caveats are in order with respect to the example in this paper. First, the example has illustrated only the relief of voltage collapse problems. It can be readily extended to consider the relief of overloads. Second, the demand management program assumes a "pay per incident" strategy for contract design. Many demand management programs in current use are based on rate rebates, particularly rate rebates for demand charges, and some of them include "buy-through" features. In some cases, customers may need greater operational flexibility than is provided by a fixed price-quantity contract. In these cases, the incorporation of buy-through clauses in contracts may prove to be valuable additions to contract design. Incorporation of these features is beyond the scope of this paper.

In order to use the theory in this paper, the payment structures in these programs must be converted into equivalent pay per incident payments. In the rate rebate program, the incentives to customers are dependent on actual probability of use of the program. Thus, the features of many existing programs do not exactly match the pay per incident assumptions in the current paper. Two solutions are possible: a redesign of existing programs along the lines suggested in this paper, or the extension of the theory of this paper to these different types of payment structures. A final caveat pertains to the continuous nature of customer behavior and the absence of bounds. In reality it is possible that customers have maximum values beyond which participation is not practical (for example, to have a customer participate in an amount that exceeds the total installed demand would require the customer to install generation equipment). These limits create discontinuities in the benefit functions. The benefit functions used in this analysis have all been smooth and continuous.

This approach can be extended beyond the utility, e.g. an Independent System Operator (ISO) can apply the same methodology. Incentives, in most ISO and Transco proposals, are linked to system performance, i.e. the ISO benefits when system congestion is reduced. When congestion occurs, a loss of surplus is inevitable. In many cases the ISO will have a choice between congestion pricing, quantity rationing ("Transmission Load Relief") or the use of interruptible power contracts. In cases when spot pricing is insufficient or too slow, the ISO can rely on demand management to attain secure operations. The same techniques used to maximize the benefit 
a utility derives with voluntary incentive-compatible contracts can be used to line up the interests of customers with those of the ISO. The difference is that the criteria to derive the ISO benefit function may differ from that of a utility. This topic will be covered in a follow up paper.

\section{CONCLUSION}

Nonlinear pricing can be used as a means for extracting maximum value from demand management contracts. By using mechanism design, optimal contracts can be designed that encourage customers to voluntarily sign up for the contract that best suits their needs. It is not necessary for a utility to know in advance the type of customer it faces when designing such programs. Cost effective load relief can be a substitute for building extra generation. The paper illustrates and incorporates the importance of location into the process. In addition it creates the opportunity for new kinds of demand management programs.

\section{REFERENCES}

[1] F. L. Alvarado, I. Dobson, and Y. Hu, "Computation of closest bifurcations in power systems," IEEE Trans. on Power Systems, vol. 9, no. 2, May 1994.

[2] M. Beenstock, "Generators and the cost of electricity outages," in Energy Economics, 1991

[3] C. A. Canizares and F. L. Alvarado, "Point of collapse and continuation methods for large ac/dc systems," IEEE Trans. on Power Systems, vol. 8, no. 1, Feb. 1993.

[4] D. W. Caves, J. A. Herriges, and R. J. Windle, "The cost of electric power interruptions in the industrial sector," Land Economics, vol. 68, no. 1, pp. 49-61, 1992.

[5] P. Dasgupta, P. Hammond, and E. Maskin, "The implementation of social choice rules," Review of Economic Studies, vol. 46, pp. 185-216, 1979.

[6] H. W. Dommel and W. F. Tinney, "Optimal power flow solutions," IEEE Trans. on Power Apparatus and Systems, vol. PAS-87, no. 10, pp. 1866-1876, Oct. 1968.
[7] M. Fahriog̃lu and F. L. Alvarado, "The design of optimal demand management programs," in Proceedings of Bulk Power System Dynamics and Control IV-Restructuring, Santorini, Greece, Aug. 1998.

[8] D. Fudenberg and J. Tirole, Game Theory: The MIT Press, 1991.

[9] J. Green and J. J. Laffont, "Characterization of satisfactory mechanisms for the revelation of preferences for public goods," Econometrica, vol. 45, pp. 427-438, 1977.

[10] S. Green, I. Dobson, and F. L. Alvarado, "Sensitivity of the loading margin to voltage collapse with respect to arbitrary parameters," IEEE Trans. on Power Systems, vol. 12, no. 1, pp. 262-272, Feb. 1997.

[11] S. Greene, "Margin and sensitivity methods for security analysis of electric power systems," Ph.D. dissertation, University of Wisconsin-Madison, Department of Electrical Engineering, 1998.

[12] M. Huneault and F. D. Galiana, "A survey of the optimal power flow literature,” IEEE Trans. on Power Systems, vol. 6, no. 2, pp. 762-770, May 1991.

[13] D. M. Kreps, A Course in Microeconomic Theory: Harvester Wheatsheaf, 1990.

[14] R. Myerson, "Incentive compatibility and the bargaining problem," Econometrica, vol. 47, pp. 61-73, 1979

[15] F. C. Schweppe, M. C. Caramanis, R. D. Tabors, and R. E. Bohn, Spot Pricing of Electricity. Boston: Kluwer Academic Publishers, 1987.

Murat Fahriog̃lu (S'92) obtained the B.S. (Hon) degree in electrical engineering from Michigan State University, and the M.S. degree from the University of Wisconsin-Madison. He is currently a Ph.D. student at the University of Wisconsin-Madison in the Department of Electrical and Computer Engineering. His main interests are in Game Theory applications in power systems and more specifically in the design of demand management contracts between electric utilities and their customers to avoid forced outages.

Fernando L. Alvarado (F'93) obtained the B.S. degree from the National University of Engineering in Lima, Peru, the M.S. degree from Clarkson University, and a Ph.D. from the University of Michigan. He is currently a Professor at the University of Wisconsin-Madison in the Department of Electrical and Computer Engineering. His main interests are power systems congestion and pricing, system security, computer applications to power systems and large scale computations. 